\title{
A gain-of-function mutation in the PDGFR- $\beta$ alters the kinetics of injury response in liver and skin
}

\author{
Monika Krampert ${ }^{1}$, Carl-Henrik Heldin ${ }^{1}$ and Rainer L Heuchel ${ }^{1,2}$
}

\begin{abstract}
Platelet-derived growth factor (PDGF) isoforms stimulate cell proliferation, migration and survival. We recently generated mice carrying a gain-of-function mutation within the activation loop of PDGF $\beta$-receptor (PDGFR- $\beta$ D849N). Embryonic fibroblasts derived from these mice show elevated basal phosphorylation and altered kinetics for ligand-induced activation of PDGFR- $\beta$, as well as enhanced proliferation and migration. To investigate the effect of this mutation in vivo, we used carbon tetrachloride-induced liver injury as a model system. We observed a higher basal activation of mutant PDGFR- $\beta$ in unchallenged livers; however, the difference in activation upon carbon tetrachloride stimulation was lower than expected, an effect that might be explained by a delayed response of the mutated receptor toward reactive oxygen species. Mutant mice showed enhanced proliferation of nonparenchymal liver cells and activation of hepatic stellate cells, leading to a small increase in early fibrosis formation. Another mouse strain lacking the binding site for phosphatidylinositol-3' kinase in PDGFR- $\beta$ showed the reverse phenotype. These results suggest an important role for PDGFR- $\beta$ signaling in the early injury-response. We confirmed this hypothesis with a second injury model, cutaneous wound healing, where we observed earlier proliferation and formation of granulation tissue in D849N-mutant mice.

Laboratory Investigation (2008) 88, 1204-1214; doi:10.1038/labinvest.2008.81; published online 1 September 2008
\end{abstract}

KEYWORDS: hyperactive PDGFR signaling; mouse models; wound healing

Members of the platelet-derived growth factor (PDGF) family promote proliferation, survival, chemotaxis and differentiation mainly of mesenchymal cells, such as fibroblasts, smooth muscle cells, pericytes and hepatic stellate cells. PDGF release is strongly enhanced in response to tissue damage, and this growth factor is thought to be involved in a variety of pathologic conditions like cancer, atherosclerosis and fibrosis (reviewed in refs. 1 and 2). PDGF homo- or heterodimers bind to two structurally related receptors, PDGFR- $\alpha$ or PDGFR- $\beta$. Upon ligand binding, receptors dimerize, resulting in activation of the intrinsic tyrosine kinase activity and autophosphorylation of the receptor. Certain of the specifically phosporylated tyrosine residues then serve as docking sites for various Src homology domain-containing proteins, initiating downstream signaling. ${ }^{3,4}$

Knockout studies revealed the importance of PDGFR- $\beta$ signaling in embryonic development, particularly in vasculogenesis and angiogenesis, as both Pdgfb and Pdgfrb knockout mice die perinatally due to widespread hemorrhages and edema formation. ${ }^{5,6}$ However, the lethality of these mice made it difficult to study the role of endogenous
PDGF signaling under normal physiologic as well as under pathologic conditions in the adult organism. The mechanisms of PDGFR activation and the contribution of the many different downstream signaling pathways to the cellular effects of PDGF have been studied in great detail using various in vitro systems (reviewed in ref. 4). However, much less is known about the importance of single downstream pathways in more complex in vivo scenarios.

We recently reported mice carrying a point mutation in the activation loop of PDGFR- $\beta$ (D849N). ${ }^{7}$ In embryonic fibroblasts derived from these mice, this mutation causes an elevated basal activation of the receptor in the absence of ligand, as well as changes in the kinetics of receptor activation and the activation of downstream signaling pathways upon PDGF-BB stimulation. As a consequence, these cells showed ligand-independent migration and enhanced proliferative response to PDGF-BB stimulation. ${ }^{7}$ In the present study, we aimed to investigate whether the effects observed in vitro are retained within a complex organism, particularly under pathologic conditions, where a multitude of different factors and signaling pathways are acting together simultaneously.

${ }^{1}$ Ludwig Institute for Cancer Research, Uppsala University, Uppsala, Sweden and ${ }^{2}$ Department of Clinical Science, Intervention and Technology, Karolinska University Hospital, Huddinge, Sweden

Correspondence: Dr RL Heuchel, PhD, Karolinska Hospital Huddinge, KFC, Center For Clinical Research, Novum, 4 th floor, 14186 Huddinge, Sweden.

E-mail: rainer.heuchel@ki.se

Received 13 December 2007; revised 30 July 2008; accepted 30 July 2008 
For that purpose, we explored two different well-established disease models, carbon tetrachloride-induced liver injury and cutaneous wound healing, as readout systems. In both models, a role of PDGF has been described in various studies (reviewed in refs. 8 and 9).

We observed changes in the activation kinetics of the mutated PDGFR- $\beta$ in the in vivo models, which correlate well with altered kinetics of the injury response. However, we also show that the activation mechanism of PDGFR- $\beta$ seems to be more complex in response to injury, particularly in the $\mathrm{CCl}_{4}$ model, compared to ligand-induced activation in vitro.

\section{MATERIALS AND METHODS Animals}

Mice carrying either an activating mutation (D849N) or a nonfunctional mutation of the binding sites for PI3K (Y739F and Y750F, in the following called $\triangle \mathrm{PI} 3 \mathrm{~K}$ ) in the PDGFR- $\beta$ have been described recently. ${ }^{7,10}$ All mice were on identical C57Bl/6 background. Only age- and sex-matched animals were used for direct comparison. All animal experiments were approved by the Local Ethical Committee.

\section{Cell Culture}

Mouse embryonic fibroblasts (MEFs) derived from D849Nmutant embryos have been described earlier. ${ }^{7}$ The cells were grown in Dulbecco's modified Eagle's medium (Sigma) supplemented with $10 \%$ fetal bovine serum and $0.1 \mathrm{mM} \beta$-mercaptoethanol. For stimulation, serum-starved cells $(0.05 \%$ FCS for $24 \mathrm{~h})$ were treated either with $500 \mu \mathrm{M}$ $\mathrm{H}_{2} \mathrm{O}_{2}$ (Merck) or $20 \mathrm{ng} / \mathrm{ml}$ PDGF-BB (kindly provided by Creative Biomolecules) for the indicated time-periods. All experiments were performed with two independent cell clones.

\section{$\mathrm{CCl}_{\mathbf{4}}$ Treatment and Induction of Fibrosis}

Mice (3-6 months old) received injections of $\mathrm{CCl}_{4}(1 \mathrm{~g} / \mathrm{kg}$ body weight, diluted 1:4 in mineral oil) intraperitoneally either as a single injection for acute liver injury studies or twice weekly over 2-4 weeks for the induction of chronic injury. Control animals received corresponding amounts of mineral oil. After the indicated time points, mice were killed and liver tissue was either frozen in liquid nitrogen or fixed overnight in $95 \%$ ethanol $/ 1 \%$ acetic acid.

\section{Skin Wounding and Preparation of Wound Tissue}

Mice (2-4 months old) were anesthetized by intraperitoneal injection of avertin $(2.5 \%$ in $0.15 \mathrm{M} \mathrm{NaCl}, 0.02 \mathrm{ml} / \mathrm{g}$ body weight). Two full-thickness excisional wounds, $5 \mathrm{~mm}$ in diameter, were made on either side of the dorsal midline by excising skin and panniculus carnosus with a biopsy punch. Wounds were left uncovered and harvested at different time points after injury. For histological analysis, the complete wounds were isolated, bisected and either directly embedded in tissue-freezing medium without prior fixation or fixed overnight in $95 \%$ ethanol $/ 1 \%$ acetic acid or $4 \%$ paraformaldehyde.

\section{Histology}

Fixed tissues were embedded in paraffin. Sections $(3 \mu \mathrm{m}$ for liver, $7 \mu \mathrm{m}$ for skin wounds) were stained with hematoxylin/ eosin for routine examination and with Sirius red to visualize collagen deposition. Morphometric analyses (necrosis, collagen deposition and wound areas) were performed using the ImageJ software (NIH). All values presented are means of 8-12 liver lobes or wounds, derived from 4 to 5 animals per data point. Statistical analysis was performed using the GraphPad Prism4 software. Results are presented as mean \pm s.d. and were analyzed by Student's $t$-test. $P \leq 0.05$ was taken as a minimum for significance.

\section{Immunohistochemistry}

Deparaffinized sections were incubated overnight with primary antibodies against $\alpha$-smooth muscle actin (ASMA; 1:500, clone 1A4, Dako), gremlin (1:200, rabbit anti-human Gremlin, IMG-5136A, IMGENEX), elastin (1:500, Purified Rabbit Anti-Rat Elastin, no. CL55041AP, CEDARLANE Laboratories), or with FITC-coupled anti-ASMA antibody (1:500, clone 1A4, Sigma), for detection of activated hepatic stellate cells and myofibroblasts, and/or an anti-platelet/ endothelial cell adhesion molecule-1 (PECAM) antibody for staining of endothelial cells. Primary antibodies were detected using biotinylated secondary antibodies (Dako), Vectastain ABC-HRP kit (Vector Laboratories) and 3,3'-diaminobenzidine peroxidase substrate kit (Vector Laboratories).

\section{Detection of Proliferating Cells by Labeling with 5'-Bromodeoxyuridine}

Mice were injected intraperitoneally with bromodeoxyuridine (BrdU) (Sigma, $50 \mathrm{mg} / \mathrm{kg}$ in $0.15 \mathrm{M} \mathrm{NaCl}$ ) and killed $2 \mathrm{~h}$ after injection. Bisected wounds were fixed in ethanol/ acetic acid as described above. Sections were incubated with a peroxidase-conjugated monoclonal antibody directed against BrdU (Roche Diagnostics) and stained using the peroxidase substrate kit (Vector). Counterstaining was performed with hematoxylin.

\section{Immunoprecipitation and Immunoblotting}

For immunoblotting, cells or tissues were homogenized in urea-buffer (10 mM Tris ( $\mathrm{pH} 8.0$ ), 9.5 M urea, $2 \mathrm{mM}$ EDTA, $1 \mathrm{mM}$ sodium vanadate, $1 \mathrm{mM}$ phenylmethyl sulfonyl fluoride) and cleared by centrifugation. Proteins were separated by SDS-polyacrylamide gel electrophoresis under reducing conditions and transferred to nitrocellulose filters. Antibody incubations were performed in $5 \%$ bovine serum albumin in Tris-buffered saline. Antibodies against phospho-Akt, Akt, phospho-p38 and phospho-ERK1/2 were obtained from Cell Signaling Technology, and an antibody against phospho-Tyr (PY99) was from Santa Cruz Biotechnology Inc. The 
antibody against the $\mathrm{C}$ terminus of PDGFR- $\beta$ was described earlier. ${ }^{11}$ For immunoprecipitation (IP) tissues were homogenized in IP-buffer $(100 \mathrm{mM}$ Tris/ $\mathrm{HCl}(\mathrm{pH} 7.5), 1 \%$ Triton $\mathrm{X}-100,5 \mathrm{mM}$ EDTA, $150 \mathrm{mM} \mathrm{NaCl}, 1 \mathrm{mM} \mathrm{Na} \mathrm{NO}_{4}, 1 \mathrm{mM}$ pefabloc and $1 \%$ trasylol). Lysates were cleared by centrifugation and preincubation with Protein A-sepharose beads. PDGFR- $\beta$ was precipitated using $2 \mu \mathrm{g}$ antibody and $20 \mu$ l Protein A-sepharose beads. Beads were washed $3 \times$ with IP-buffer and eluted with $2 \times$ Laemmli sample buffer. Samples were analyzed by immunoblotting, as described above.

\section{RESULTS}

The D849N Mutation in the PDGFR- $\beta$ Alters the Kinetics of Receptor-Phosphorylation and Downstream Signaling in Response to Carbon Tetrachloride-Induced Liver Injury

We recently showed that introduction of a point mutation (D849N) into PDGFR- $\beta$ results in dramatically changed kinetics of receptor activation following ligand stimulation of MEFs. To investigate if this mutation has similar consequences in more complex in vivo situations, we explored the model of $\mathrm{CCl}_{4}$-induced liver injury in our mutant mice, a system where induction of PDGF and PDGF receptors has been described in several studies (reviewed in Bonner ${ }^{8}$ ). $\mathrm{CCl}_{4}$ $(1 \mathrm{~g} / \mathrm{kg})$, or mineral oil as control, was injected i.p. and tissue samples taken at a series of time points. Tissue lysates were analyzed for activation of PDGFR- $\beta$ by immunoprecipitation of the wild type and mutant receptor and detection of phosphorylated tyrosine residues by western blotting. Lysates from PDGF-BB stimulated fibroblasts served as a positive control. Activation of known downstream targets of PDGFR- $\beta$ was analyzed using a series of phospho-specific antibodies.

As seen in Figure 1, $\mathrm{CCl}_{4}$ caused a strong phosphorylation of PDGFR- $\beta$ as well as activation of Erk1/2, Akt and p38 already $10-20 \mathrm{~min}$ after application in wild-type mice. Interestingly, the basal activity of the receptor was markedly higher in liver tissue of mice carrying the mutant PDGFR- $\beta$, an observation that had already been made in MEF cells derived from these animals. ${ }^{7}$ This confirmed that the effect of the mutation seen in vitro is not compensated for in vivo. Unexpectedly, further receptor-activation in response to the injury occurred slightly slower in mutant mice compared to wild type (Figure 1a). A very similar pattern was seen with regard to the activation of Erk1/2, a downstream signaling component in the PDGFR signaling pathway. In contrast, for Akt and p38 the most striking difference was the high basal phosphorylation in the mutant mice, whereas after injury the phosphorylation initially decreased and never exceeded the elevated basal level or the activation level observed in wild-type mice (Figure 1c).

\section{Fibroblasts Carrying the PDGFR- $\beta$ D849N Mutation} Respond More Slowly to Hydrogen Peroxide-Treatment

Carbon tetrachloride exerts its toxic effect toward liver tissue mainly by the generation of various highly reactive radicals. ${ }^{12}$
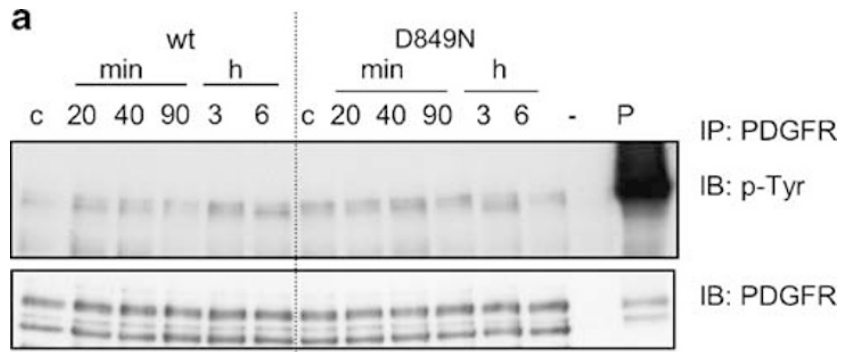

IB: PDGFR
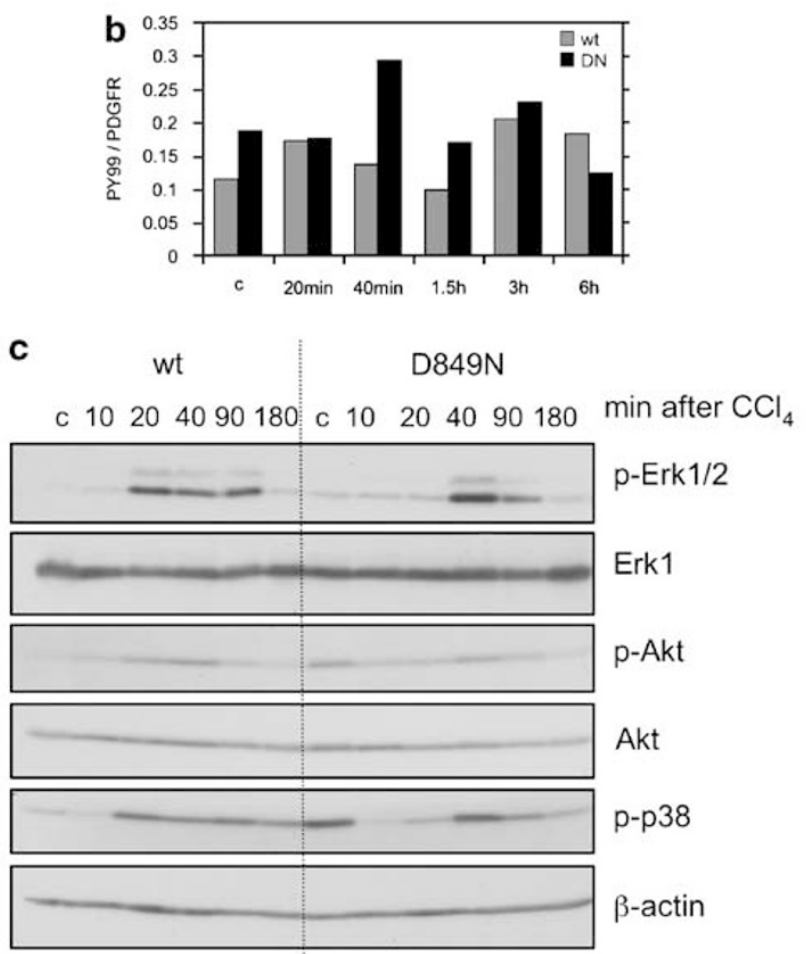

Figure 1 Altered kinetics of PDGFR- $\beta$ activation and downstream signaling in $\mathrm{D} 849 \mathrm{~N}$-mutant mice in response to $\mathrm{CCl}_{4}$-induced liver injury. Wild-type (wt) or D849N-mutant (DN) mice were injected with $1 \mathrm{~g} / \mathrm{kg} \mathrm{CCl}_{4}$ in mineral oil, or mineral oil as control (c) and killed at the indicated time points. (a) PDGFR- $\beta$ was immunoprecipitated from liver lysates (IP). Precipitated proteins were analyzed by immunoblotting (IB) using anti-phosphotyrosine (PY99; upper panel) and anti-PDGFR- $\beta$ (lower panel) antibodies. (b) Densitometric quantification (using Image J software) of receptor phosphorylation and normalization to the total receptor levels. (c) Tissue lysates ( $80 \mu \mathrm{g}$ of total protein) were analyzed by immunoblotting for the activation of Erk1/2, Akt and p38, using phospho-specific antibodies. Levels of total Erk1, total Akt and $\beta$-actin were determined as loading controls. As positive control ('P'), MEF cells were treated with $20 \mathrm{ng} / \mathrm{ml}$ PDGF-BB for $5 \mathrm{~min}$. One representative result is shown out of a total of three independent experiments.

Recently, it had been shown that PDGFR can be activated directly by reactive oxygen species (ROS), such as hydrogen peroxide in a ligand-independent mechanism. ${ }^{13,14}$ To investigate whether the D849N mutation also affects this activation mode, we stimulated MEF cells derived from PDGFR- $\beta$ D $849 N$-mutant mice and corresponding controls with $\mathrm{H}_{2} \mathrm{O}_{2}$. To stay close to a system of ROS-mediated 
toxicity, we choose $\mathrm{H}_{2} \mathrm{O}_{2}$ concentrations that had been reported to be cytotoxic for fibroblasts upon prolonged exposure. ${ }^{15}$ Stimulation with PDGF-BB was used as positive control. Interestingly, D849N-mutant cells showed a strongly delayed activation of PDGFR- $\beta$ compared to the wild-type controls (Figure 2a), similar to that observed in vivo; whereas in the wild-type cells the maximum of receptor activation was reached already after $5 \mathrm{~min}$, it took $40 \mathrm{~min}$ to get full activation for the mutant cells. To a lower extent, this delayed kinetics was also observed for activation of some of PDGFR- $\beta$ 's downstream targets, such as phosphorylation of Erk-1/2 and Akt (Figure 2c). In contrast to the complex in vivo situation shown in Figure 1c, the enhanced basal activation of the downstream effectors in cells carrying the mutant receptor was less pronounced in the cell culture experiments, despite the clear basal activation of the mutant receptor (Figure $2 \mathrm{a}$ and $\mathrm{c}$ ) and only visible after prolonged exposure.

\section{The PDGFR- $\beta$ D849N Mutation Causes Enhanced Proliferation and Activation of Hepatic Stellate Cells after Acute Injury}

To examine potential consequences of the altered kinetics of PDGFR- $\beta$ activation after $\mathrm{CCl}_{4}$ injury on the healing response, we measured the proliferation rate of nonparenchymal cells (the expression site of PDGFR- $\beta$ in the liver) at different time points after injury. As revealed by BrdU-incorporation, mutant mice showed significantly higher DNA synthesis $60 \mathrm{~h}$ after $\mathrm{CCl}_{4}$ administration (Figure 3a), the time point where both control and mutant mice reached the maximal rate of proliferation. No difference was seen in unchallenged livers and after $48 \mathrm{~h}$, whereas after $36 \mathrm{~h}$ mutant mice showed slightly lower BrdU incorporation. No significant difference was seen in the proliferation of hepatocytes (data not shown). The distribution of BrdU-positive cells over the liver was similar in both genotypes: $36 \mathrm{~h}$ after injury, BrdU-positive cells were mainly found in the periportal area; no BrdU-positive cells were found in the necrotic areas. At $48 \mathrm{~h}$, the first nonparenchymal BrdU-positive cells were detected also in the necrotic areas and $60 \mathrm{~h}$ after $\mathrm{CCl}_{4}$ administration, high numbers of proliferating cells were distributed all over the liver, with hepatocytes still restricted to the undamaged areas.

As a second readout of the injury response, we examined the activation of fibrogenic cells by immunostaining for the marker ASMA. ASMA-positive cells comprise the smooth muscle cells in the wall of the portal vein and artery, myofibroblasts of the portal and pericentral area and activated hepatic stellate cells along the sinusoids. ${ }^{16,17}$ In controlinjected animals, only the linings of the vessel walls (portal and central) stained positive for ASMA. The first activated fibrogenic cells were detected in the pericentral area $48 \mathrm{~h}$ after injury (Figure 3b, upper panel). In some of the mutant samples, ASMA staining seemed stronger than in the controls, but did not reach statistical significance, due to the high inter-individual differences. At $60 \mathrm{~h}$ after $\mathrm{CCl}_{4}$ injection,
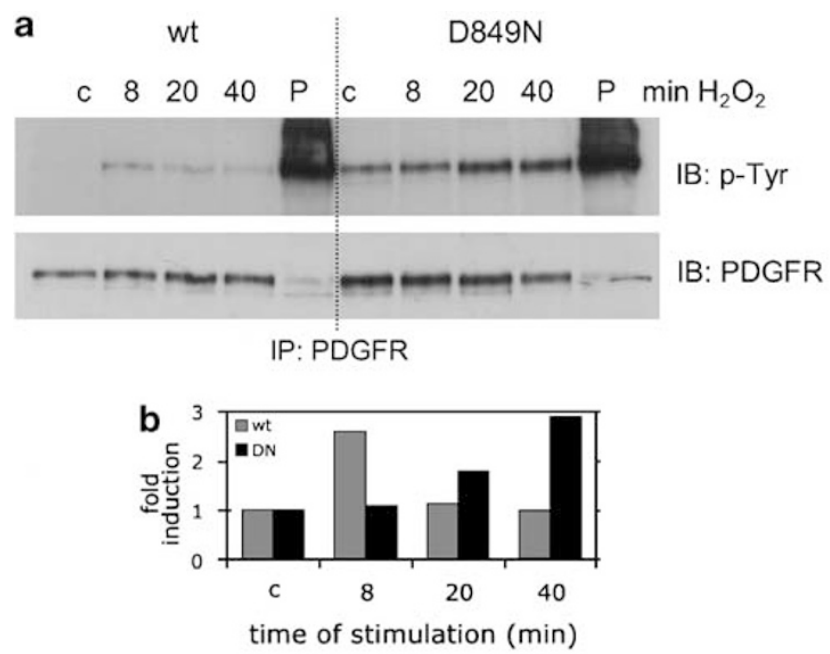

C

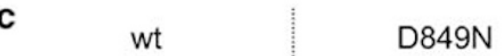

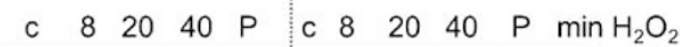

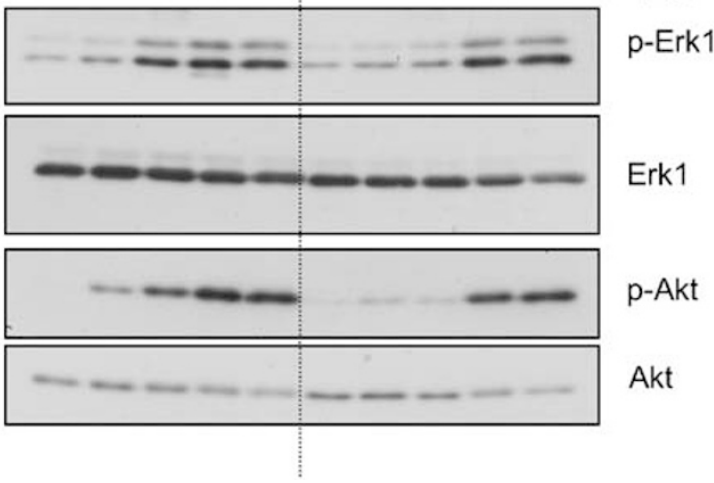

Figure 2 Delayed response to $\mathrm{H}_{2} \mathrm{O}_{2}$ in D849N-mutant MEF cells. Wild-type or D849N-mutant MEF cells were serum starved and subsequently stimulated with $500 \mu \mathrm{M} \mathrm{H}_{2} \mathrm{O}_{2}$ for the time indicated. Stimulation with $20 \mathrm{ng} / \mathrm{ml}$ PDGF-BB ( $5 \mathrm{~min}$ ) was used as a positive control ('P'). (a) PDGFR- $\beta$ was immunoprecipitated from cell lysates (IP). Precipitated proteins were analyzed by western blotting (IB) using anti-phospho-tyrosine (upper panel) and anti-PDGFR- $\beta$ (lower panel) antibodies. (b) Densitometric quantification of the receptor phosphorylation and normalization to the total receptor levels. (c) Cell lysates $(20 \mu \mathrm{g})$ were analyzed by western blotting for the activation of Erk1/2, Akt and p38, using phospho-specific antibodies. Levels of total Erk1, total Akt and $\beta$-actin were determined as loading controls. Total p38 was omitted, as it was below the detection level of the antibody. One representative result is shown out of a total of three or more independent experiments.

ASMA-positive cells had spread widely around from the central areas, whereas in the portal tract, staining was restricted to the vessel walls. Morphometric measurements revealed significantly stronger ASMA staining, and thus HSC activation, in mutant compared to wild-type animals (Figure 3c).

To exclude that the observed increase in proliferation and HSC activation was a consequence of potentially higher sensitivity toward $\mathrm{CCl}_{4}$ and subsequently larger tissue damage rather than stronger PDGFR activation, we measured 

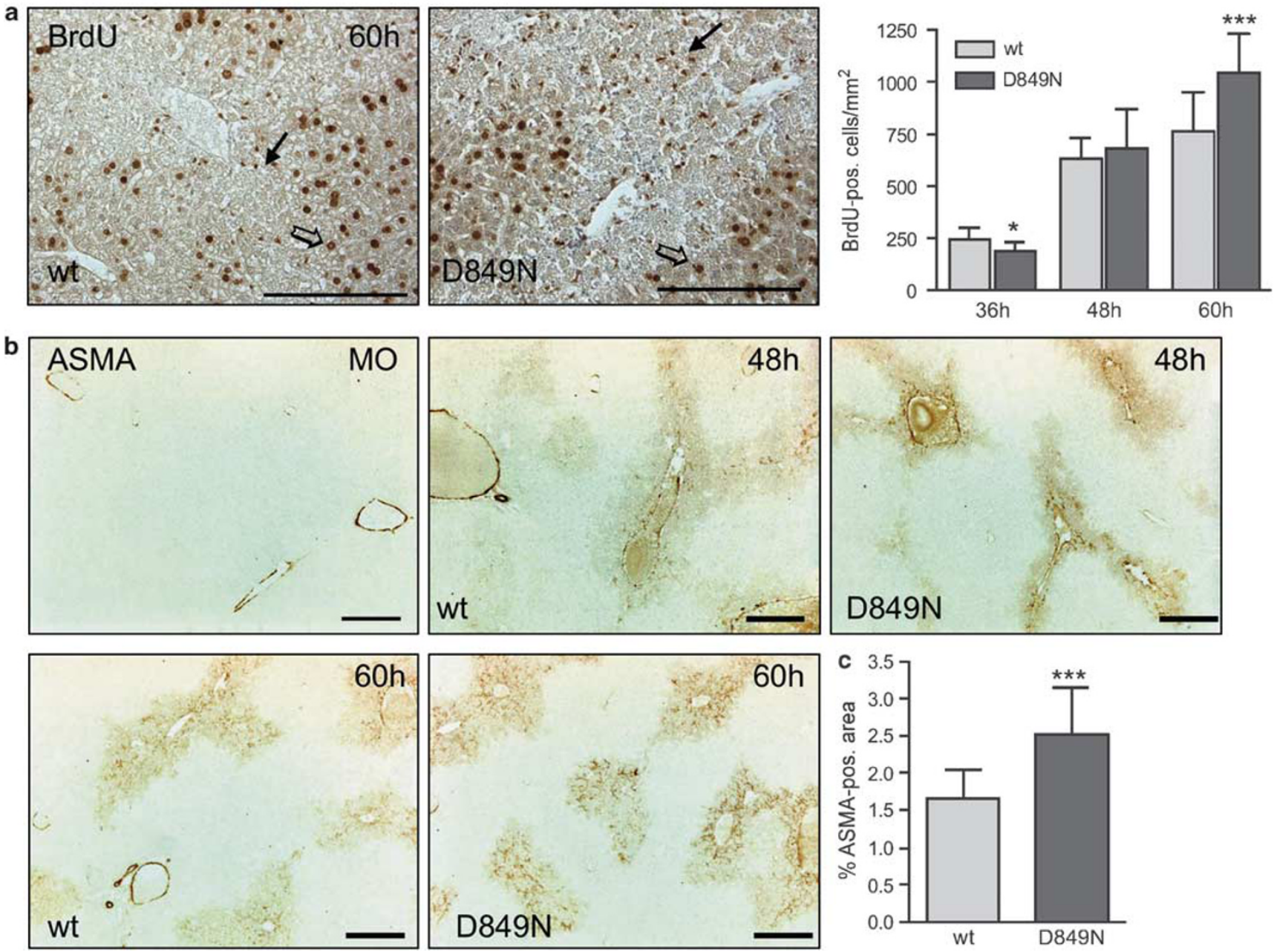

Figure 3 Enhanced proliferation and fibrogenic cell activation in D849N-mutant mice after $\mathrm{CCl}_{4}$-induced acute liver injury. Wild-type (wt) or D849N-mutant (DN) mice were injected with $1 \mathrm{~g} / \mathrm{kg} \mathrm{CCl}_{4}$ or mineral oil as control (MO) and killed after 36, 48 or $60 \mathrm{~h}$. (a) Analysis of the proliferation rate of nonparenchymal cells. Mice were injected with $50 \mathrm{mg} / \mathrm{kg}$ BrdU $2 \mathrm{~h}$ before the end of the experiment. Sections from ethanol-fixed, paraffin-embedded tissue derived from wild-type (left) and D849N-mutant mice (middle) (5 mice per time point and genotype) were stained with anti-BrdU antibody (dark brown signal) and positive cells counted using Image J software. Hepatocytes (open arrow) and nonparenchymal cells (filled arrow) were distinguished morphologically. To the right, results for nonparenchymal cells are shown. Statistical analysis was performed using Student's $t$-test, $P$-values are $P=0.021$ for $36 \mathrm{~h}, P=0.447$ for $48 \mathrm{~h}$ and $P=0.0004$ for $60 \mathrm{~h}$. (b, c) Analysis of cell activation. Sections from the same samples were stained with anti-ASMA antibody (dark brown signal). (b) Representative mineral oil-treated control (MO), $\mathrm{CCl}_{4}$ treatment of wild-type and D849N-mutant mice for 48 and $60 \mathrm{~h}$. Positive stained areas were quantified morphometrically and presented as percent of the total image area. Results are shown in $(\mathbf{c}), P=0.0015$ for the $60 \mathrm{~h}$ time point. Scale bars: $100 \mu \mathrm{m}, \mathrm{PV}$ : portal vein, CV: central vein.

necrotic areas within the livers on histologic sections; however, no difference in the amount of necrosis was found at any of the time-points analyzed (data not shown).

\section{The Fibrotic Response to Chronic $\mathrm{CCl}_{4}$ Injury is Transiently Increased in PDGFR- $\beta$-D849N-Mutant Mice}

Chronic liver injury, experimentally modeled by repeated administration of $\mathrm{CCl}_{4}$, causes development of fibrosis. To investigate if the enhanced activation of hepatic stellate cells we observed after acute injury also leads to a stronger fibrotic response, we treated mice twice a week with $\mathrm{CCl}_{4}(1 \mathrm{~g} / \mathrm{kg}$ body weight) for 2 and 4 weeks. This setup allowed us to monitor both the onset of collagen deposition (2 weeks) and to quantify established fibrosis (4 weeks). Using picrosirius red staining to visualize deposited collagen fibers, we observed a slight, but statistically significant, increase in collagen deposition in the D849N-mutant animals after 2 weeks of treatment (Figure 4a). In contrast, after 4 weeks there was no more difference between wild-type and mutant mice (Figure $4 \mathrm{~b}$ ), indicating that this PDGFR- $\beta$ mutation mainly affects the acute injury response and the onset of fibrotic disease, but is less important for the later stages of fibrosis.

Besides hepatic stellate cells, also myofibroblasts and portal fibroblasts have been reported to participate in the fibrotic 
a

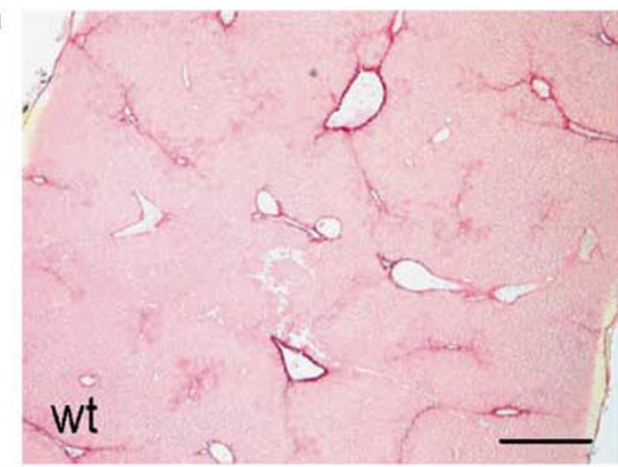

b

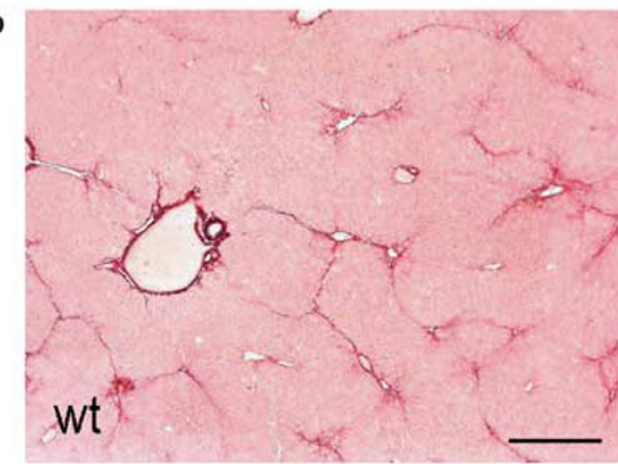

C

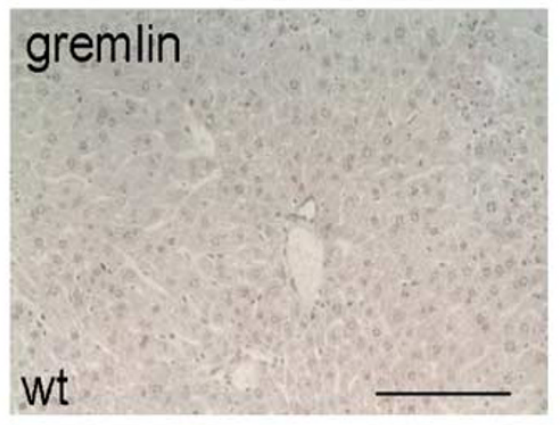

\section{D849N}
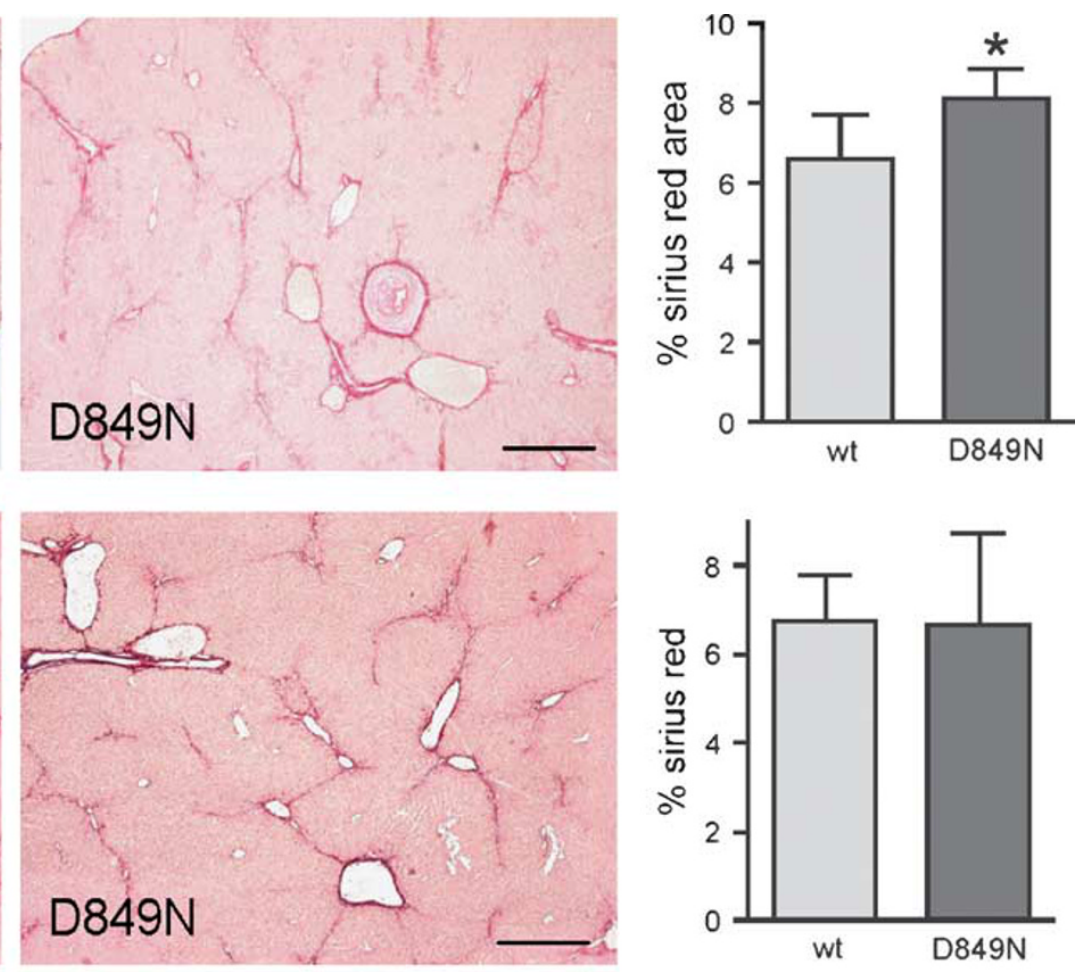

2 weeks
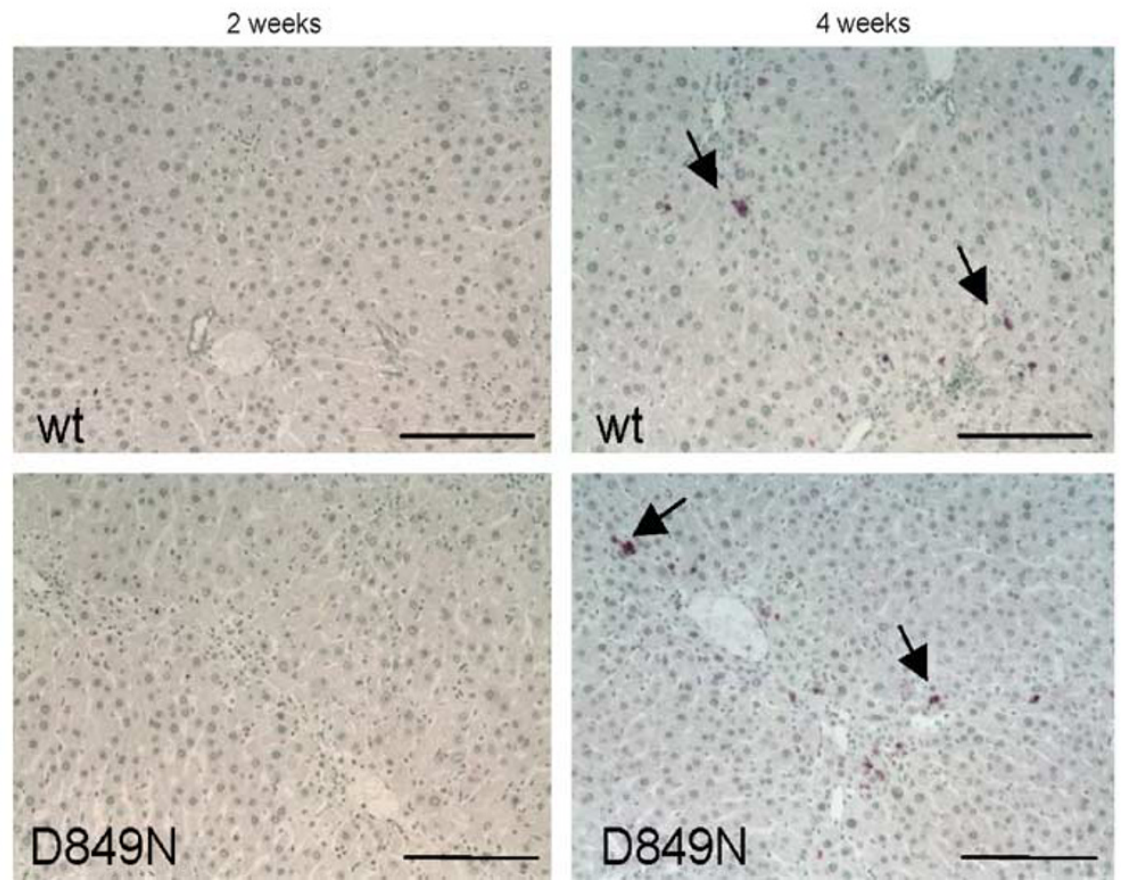

Figure 4 Slightly enhanced fibrosis in D849N-mutant mice. Mice were treated with $1 \mathrm{~g} / \mathrm{kg} \mathrm{CCl}_{4}$ twice weekly for 2 weeks (a) or 4 weeks (b). Fibrosis was analyzed by staining sections from wild-type (left panels) and D849N-mutant (middle panels) animals with picrosirius red. Stained areas were quantified and presented as percent of total tissue area (right panel). See legend to Figure 3 for details on statistical analysis. $P$-values are $P=0.032$ for 2 weeks and $P=0.948$ for 4 weeks. Scale bars: $200 \mu \mathrm{m}$. (c) IHC-staining for the myofibroblast marker gremlin in sections from wild-type (upper panel) or D849N-mutant (lower panel) mice, treated with $\mathrm{CCl}_{4}$ for $60 \mathrm{~h}$ (left panel), 2 weeks (middle panel) or 4 weeks (right panel) as described above. Scale bars: $100 \mu \mathrm{m}$.

response. ${ }^{18-20}$ Recently, the secreted protein gremlin has been identified as a specific marker for myofibroblasts. ${ }^{17}$ We analyzed protein expression of gremlin in sections from mice induced with $\mathrm{CCl}_{4}$ for $60 \mathrm{~h}, 2$ and 4 weeks to further characterize the fibrotic response in our mice (Figure 4c). We found gremlin-positive cells along the liver sinusoids in mice 
a
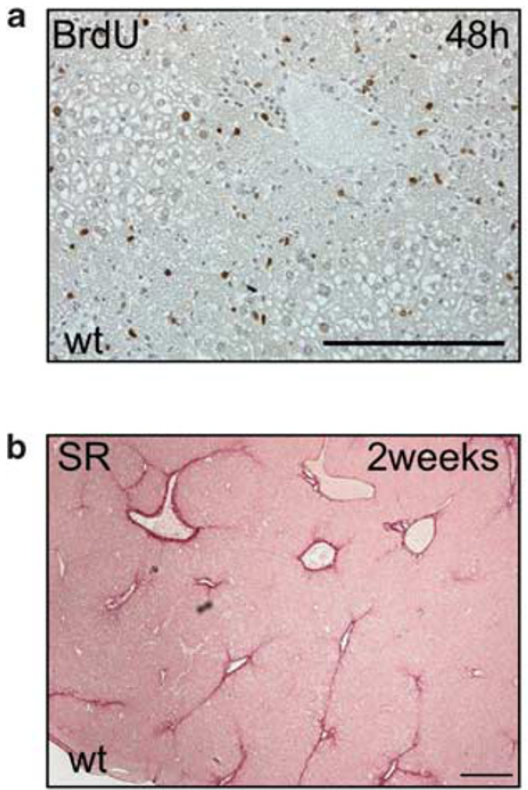
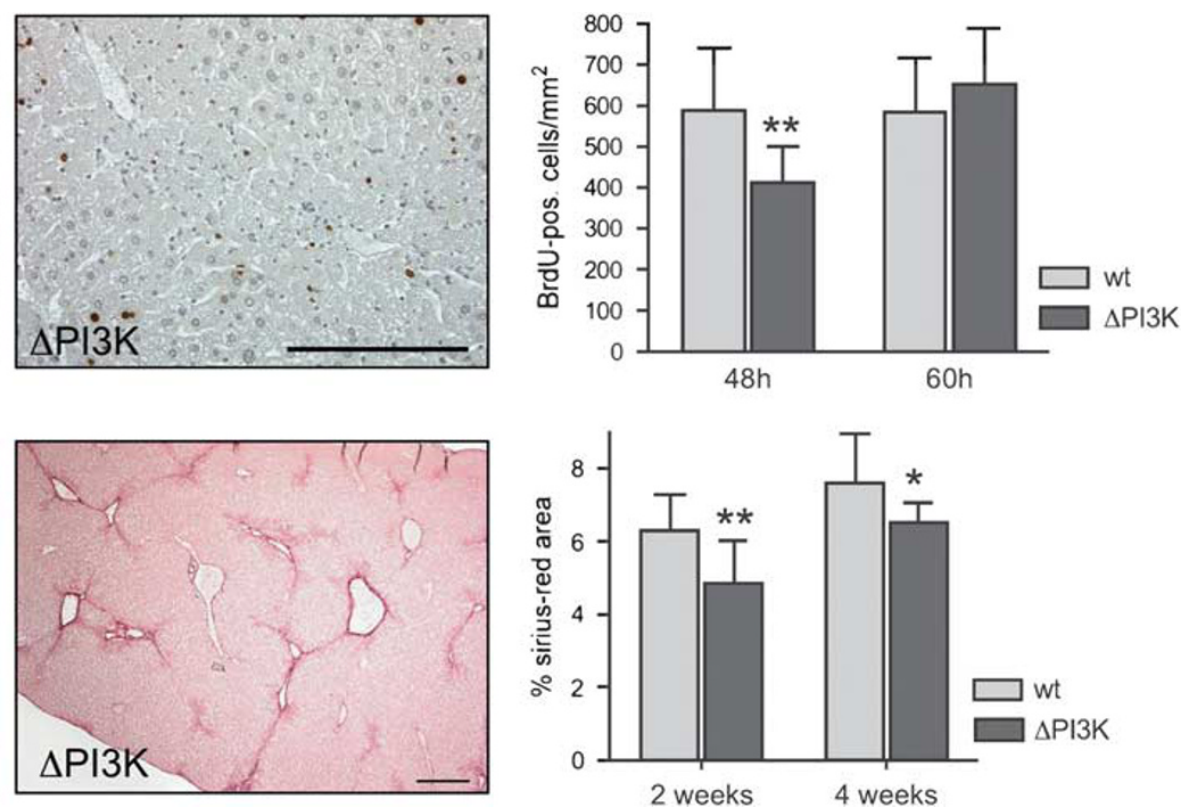

Figure 5 Reduced injury response in PDGFR- $\beta$ - $\Delta$ PI3K-mutant mice. (a) Wild-type (left panel) and PDGFR- $\beta$ - $\Delta$ PI3K-mutant (middle) mice received a single injection of $1 \mathrm{~g} / \mathrm{kg} \mathrm{CCl}_{4}$ and were killed after 48 and $60 \mathrm{~h}$. Proliferation was measured by BrdU incorporation as described in the legend to Figure 3 . Pictures show staining of $48 \mathrm{~h}$ sections. Quantification is shown to the right, $P=0.0014$ for $48 \mathrm{~h}$ and $P=0.217$ for $60 \mathrm{~h}$. (b) Wild-type (left panels) and PDGFR- $\beta$ - $\Delta$ PI3Kmutant (middle) mice were treated with $1 \mathrm{~g} / \mathrm{kg} \mathrm{CCl}_{4}$ for 2 (left and middle panel) or 4 weeks. Fibrosis was analyzed by staining with picrosirius red and quantified as described in Figure 4. The right panel shows results for 2 and 4 weeks. $P=0.0031$ for 2 weeks, $P=0.019$ for 4 weeks. Scale bars: $100 \mu$ m.

treated with $\mathrm{CCl}_{4}$ for 4 weeks, but not at earlier time points. No significant difference was found between wild-type and mutant animals (data not shown), in accordance with the observation that collagen deposition was no longer elevated in mutant mice at this time point. Similar results were obtained for the portal tract fibroblast marker elastin (data not shown). Therefore, we conclude that in our model, pericentral hepatic stellate cells are the major cell type affected by the D849N mutation, which is in accordance with both the strong expression of the PDGF receptor in these cells ${ }^{8}$ and the fact that $\mathrm{CCl}_{4}$ is predominantly a centrilobular toxin. ${ }^{21}$

\section{Mutant Mice Lacking the Binding Site for PI3-Kinase in the PDGFR- $\beta$ Show Reduced Proliferation after Acute Injury and Less Collagen Deposition after Chronic $\mathrm{CCl}_{4}$ Administration}

Observations from other groups have shown a pivotal role of PI3 kinase in mediating the effects of PDGF on proliferation and migration of hepatic stellate cells. ${ }^{22}$ We recently established mutant mice lacking the binding site for PI3' kinase in the PDGFR- $\beta$ (called ' $\Delta$ PI $3 K^{\prime}$ '), thus eliminating the activation of PI3K in response to PDGF and certain downstream signaling pathways, such as the Akt pathway. ${ }^{10}$ In several aspects, MEF cells derived from these mice show the opposite phenotype compared to cells carrying the D849N mutation of the PDGFR- $\beta$, such as reduced chemotaxis in response to the ligand. Therefore, we used these mice as a complementary model to investigate the effects of altered PDGFR- $\beta$ signaling on injury response in the liver and onset of fibrotic disease.
Upon $\mathrm{CCl}_{4}$-induced liver injury, we observed a delayed proliferative response in the $\Delta \mathrm{PI} 3 \mathrm{~K}$ mice compared to the wild type, with a significantly reduced number of BrdUpositive cells after $48 \mathrm{~h}$ (Figure $5 \mathrm{a}$ ). After $60 \mathrm{~h}$, the mutant mice had reached the proliferation level of the wild-type mice, indicating a shift in the injury-response kinetics rather than generally impaired proliferation.

Interestingly, this delayed response toward $\mathrm{CCl}_{4}$ resulted in reduced collagen deposition, as revealed by Sirius red staining. This effect was more pronounced after 2 weeks of chronic $\mathrm{CCl}_{4}$ application, but still statistically significant after 4 weeks (Figure 5b).

\section{Reformation of Dermal Tissue after Skin Wounding is Accelerated in Mice Carrying the Activating D849N Mutation in the PDGFR- $\beta$}

To confirm the effect of a mutated PDGFR- $\beta$ on the kinetics of injury response in a second wounding model, we performed excisional skin wounding experiments with the D849N mice. Release of PDGF by degranulating platelets is a very early event after wounding, ${ }^{9}$ therefore we focused mainly on the early phase of the healing response. Histological examination of wounds at days 3 and 5 indeed showed significantly enlarged areas of granulation tissue in the mutant mice compared to the wild-type controls at both time points (Figure $6 \mathrm{a}-\mathrm{c}$ ). In accordance with this observation, we found a higher proliferation rate of dermal cells in 3-day wounds, as revealed by staining for incorporated BrdU (Figure 6d). The total number of BrdU-positive cells was 
elevated, and the area containing positive cells extended farther toward the middle of the wound. Interestingly, at day 5 we observed a lower proliferation rate in the mutant mice (Figure 6e), suggesting that they reached the peak of pro-
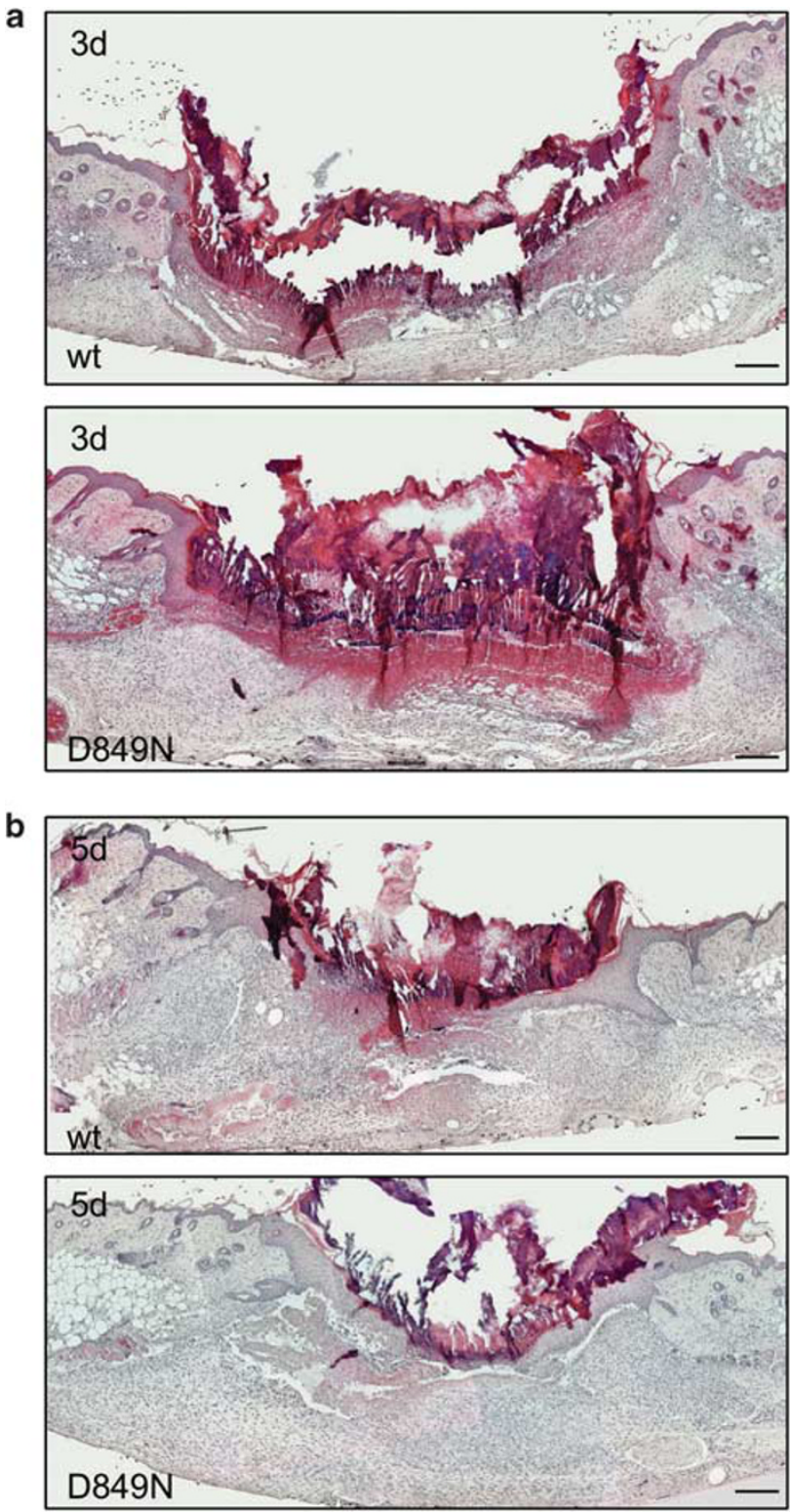

c

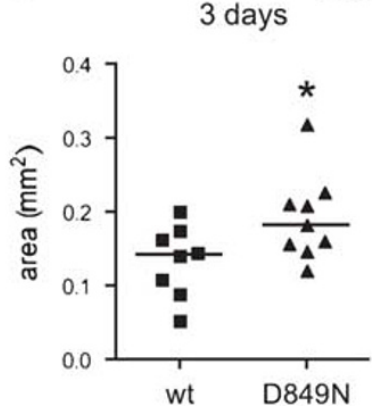

Granulation Tissue

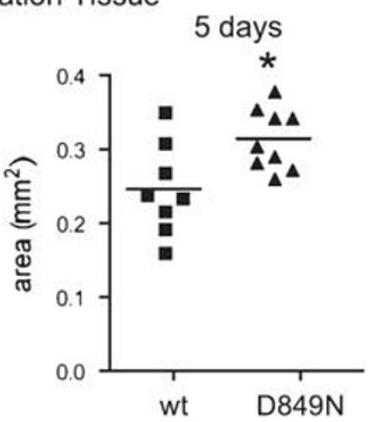

liferation earlier than wild-type mice, resulting in an earlier reformation of the dermal tissue. Staining for blood vessels using the endothelial marker PECAM revealed no differences in the revascularization of the wound (Figure 6f). PDGF is also thought to be involved in the differentiation of fibroblasts into myofibroblasts during wound healing, a process responsible for wound contraction. However, in stainings for the marker $\alpha$-smooth muscle actin, equal amounts of myofibroblasts were found in wild-type and mutant mice (Figure 6f). In accordance with this finding, also morphometric measurements did not reveal differences in wound diameter (data not shown), indicating that the mutation does not influence wound contraction. Other wound parameters like reepithelialization and wound closure were not altered either (data not shown).

\section{DISCUSSION}

Platelet-derived growth factor is a mitogenic factor involved in a large variety of physiologic and pathologic processes, such as angiogenesis, malignant transformation, fibrotic diseases and tissue regeneration. ${ }^{4}$ In the present work, we describe the effects of a gain-of-function mutation in the PDGF $\beta$-receptor observed in animal models for tissue repair processes.

Mutations of a conserved aspartate residue in the activation loop of related tyrosine-kinase receptors have been found in a number of human tumors (reviewed in Jones and $\operatorname{Cross}^{23}$ ). In particular, mutations of aspartate 842 in the highly similar PDGFR- $\alpha$ were identified in gastrointestinal stromal tumors. We therefore introduced an activating mutation of the corresponding amino-acid residue in the $p d g f r b$ gene to generate an animal model of enhanced PDGFR- $\beta$ signaling. ${ }^{7}$ This mutation alone is not sufficient to cause tumor formation, and mice also do not show abnormalities

Figure 6 Accelerated skin wound healing in D849N-mutant mice. Five mm full-thickness excisional wounds were generated on the back of wild-type (wt) and D849N-mutant (DN) mice. (a, b) Sections from the middle of 3- (a) and 5-day (b) wounds were stained with hematoxylin/eosin. (c) Area quantification of granulation tissue in 3-day (left) and 5-day- (right) old wounds using Image J software. 12-15 wounds derived from 4-5 animals were measured for each time point and genotype. Only functional new tissue was counted, whereas pure matrix deposits were neglected. $P=0.043$ for 3 -day and $P=0.016$ for 5 -day wounds (Student's $t$-test). (d, e) Analysis of the proliferation rate of cells within the granulation tissue. Mice were injected with $50 \mathrm{mg} / \mathrm{kg} \mathrm{BrdU} 2 \mathrm{~h}$ before the end of the experiment. Sections from the middle of the wounds were immunostained with antiBrdU antibody. BrdU-positive cells in the granulation tissue were counted and depicted as the number of positive cells per $1000 \mathrm{~mm}^{2}$ granulation tissue (right panel). $P=0.024$ for 3 -day and $P=0.0015$ for 5 -day wounds as revealed by Student's $t$-test. (f) Sections from 5-day wounds from wild-type (left) and mutant (right) mice were immunostained for $\alpha$-smooth muscle actin (ASMA, green) as a marker for myofibroblasts, and PECAM (red) as a marker for endothelial cells/blood vessels. The area containing myofibroblasts was measured for each wound (nine wounds per genotype, $P=0.463$ ), shown in the right panel. Scale bars: $100 \mu \mathrm{m}$. 

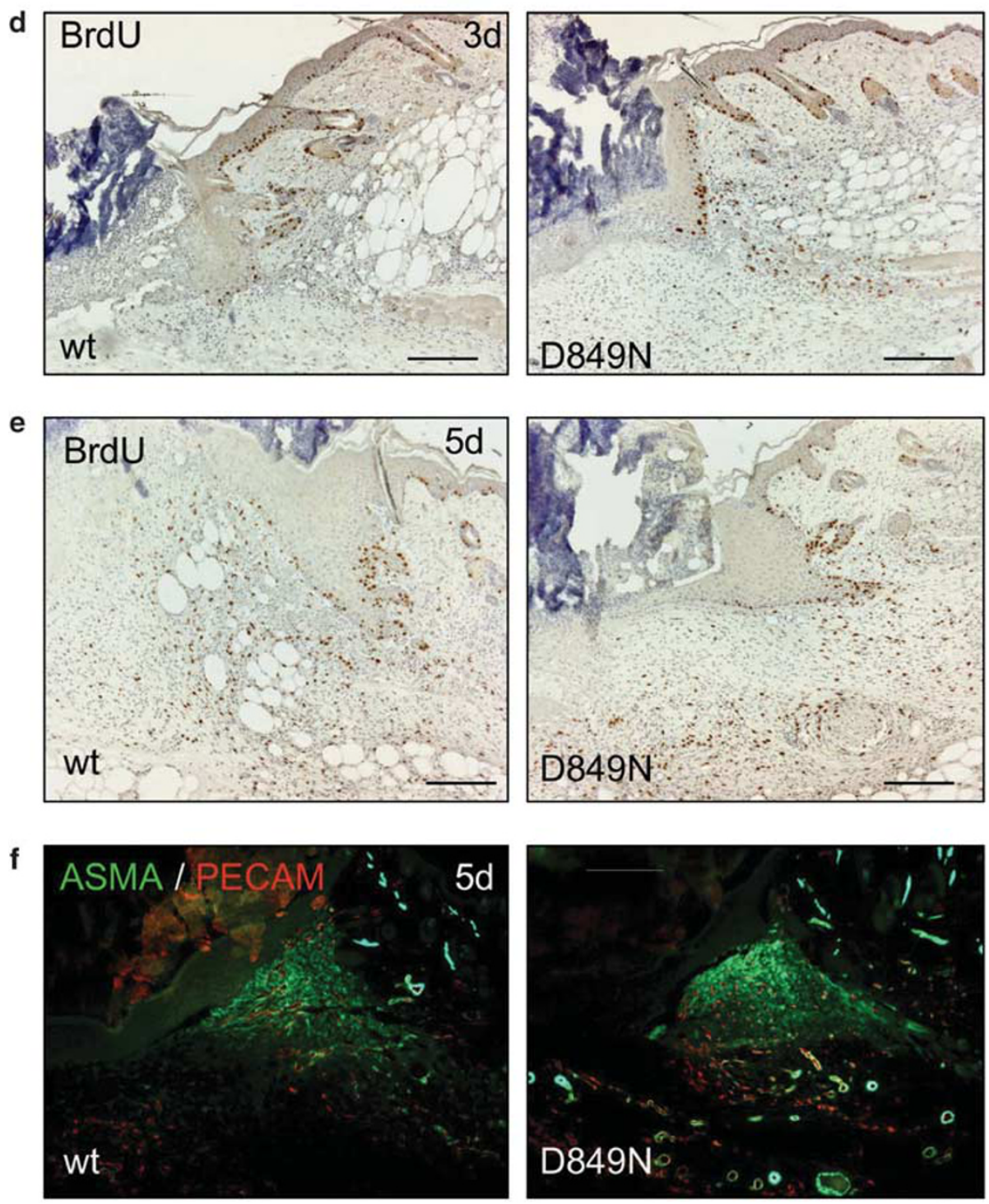

Figure 6 Continued.

in unchallenged adult animals, thus making them an excellent model for studying the effects of enhanced PDGFR signaling in disease models without interference of systemic effects.

Embryonic fibroblasts carrying the D849N mutation show ligand-independent activation of PDGFR- $\beta$, altered kinetics of PDGF-response as well as enhanced proliferation and chemotaxis. ${ }^{7}$ In this study, we investigated the effect of this mutation in vivo, where many different factors are involved simultaneously. We choose the model of $\mathrm{CCl}_{4}$-induced liver injury to monitor PDGFR- $\beta$ activation in response to tissue damage, because the role of PDGF is well established in this setup (reviewed in Bonner ${ }^{8}$ ). The most prominent difference we found in mutant animals was the enhanced basal activation of PDGFR- $\beta$ and several of its downstream effectors such as Erk1/2, Akt and p38, thus correlating well with our previous in vitro data. Interestingly, this activation did not lead to enhanced proliferation or activation of hepatic stellate cells in unchallenged animals, indicating that in vivo, either the slightly elevated activity of these pathways is not sufficient to induce HSC proliferation, or other regulatory mechanisms exist that counteract the effects of activated PDGFR- $\beta$. Against our expectations, the difference in the activation pattern between wild-type and mutant PDGFR- $\beta$ after $\mathrm{CCl}_{4}$ challenge was rather small. A notable feature was also the very early phosphorylation response, already $10-20 \mathrm{~min}$ after injury. This time course seems too fast to be mediated by release of PDGF from invading inflammatory cells or by induction and secretion of PDGF by liver cells. Both observations might be explained by the mechanism of $\mathrm{CCl}_{4}{ }^{-}$ induced liver injury: hepatotoxicity in this system is mainly mediated by the generation of radicals and the production of 
ROS. ${ }^{12}$ Recently, it has been shown that PDGFR- $\beta$, as well as other tyrosine-kinase receptors, can be activated directly by ROS. ${ }^{13,14}$ To investigate if the point mutation we introduced into the PDGFR- $\beta$ also affects its activation by reactive oxygen, we studied this process in MEF cells. Surprisingly, the mutated receptor displayed a markedly delayed response toward ROS in cell culture, in contrast to the enhanced activation by ligand. The mechanism of this effect remains to be elucidated in further studies; it is possible that conformational changes in the mutant receptor impair its immediate activation by ROS, or that the overall sensitivity of the cells toward reactive oxygen has been altered through an adaptation process. It is tempting to speculate that two different mechanisms of PDGFR- $\beta$ activation, by ROS and later by PDGF, occur during $\mathrm{CCl}_{4}$-induced injury. Most likely the activating point mutation we introduced influences both processes in opposite manners, which would explain the unexpected pattern of receptor activation we observed.

Does this altered activation pattern have any consequences for the injury response in the mutant animals? In accordance with higher receptor activation at later time points, we found elevated proliferation levels in the mutant animals $60 \mathrm{~h}$ after acute injury, but not at the onset of proliferation (36 and $48 \mathrm{~h}$ ). In a chronic liver injury model, however, we saw only a minor increase in the fibrotic reaction in the mutant animals, indicating that the hyperactive PDGFR- $\beta$ mainly affects the early proliferative response after injury and the initial activation of fibrogenic cells, whereas it has only weak influence on chronic disease.

The idea of PDGF as a factor regulating early events in the development of fibrotic disease is supported by recent studies, suggesting that other growth factors, such as TGF- $\beta$, are major players in collagen deposition and fibrosis formation during chronic liver injury (reviewed in Gressner et $a^{24}$ ). In addition, it had been shown in rodent models that PDGFR inhibitors are only effective against fibrosis if applied before injury ${ }^{25,26}$ but do not revert already existing fibrosis. ${ }^{27}$ These data are consistent with our results with mice lacking the binding site for PI3-kinase on PDGFR- $\beta$, which show a delayed proliferation in response to $\mathrm{CCl}_{4}$ injury, as well as a weaker fibrotic response. The importance of the PI3K signaling pathway for the activation of hepatic stellate cells by PDGF has been demonstrated in vitro; ${ }^{22}$ however, we show here for the first time that this pathway is also essential in vivo.

As the dual mechanism of PDGFR- $\beta$ activation by $\mathrm{CCl}_{4}$ complicated the interpretation of our results, we looked for a second injury model to better understand the effects of a hyperactive receptor on early injury response. During cutaneous wound healing, high amounts of PDGF are released by platelets during the blood clotting process immediately after the mechanic injury. ${ }^{9}$ Production of ROS during the inflammatory phase of wound healing occurs later and at relatively low amounts compared to $\mathrm{CCl}_{4}$ injury, ${ }^{28,29}$ so it is safe to assume that in the skin model PDGFR is pre- dominantly activated by its ligand. We observed that the activating mutation in the PDGFR- $\beta$ shifted the kinetic of proliferation in the dermal compartment and the formation of granulation tissue toward earlier time points. Interestingly, the proliferative phase of wound healing also ended earlier, indicating that the regulatory mechanisms that control and synchronize the healing response are not disturbed by the mutant PDGF receptor.

Much of the knowledge about PDGF actions during injury response and tissue regeneration is derived from studies that rely on overexpression or application of high amounts of exogenous PDGF. ${ }^{30,31}$ Here we show that the rather moderate increase in PDGF signaling we achieve by a single point mutation in the PDGFR- $\beta$ is sufficient to increase particularly the early proliferative phase of injury response.

\section{ACKNOWLEDGEMENTS}

We thank Aive Ahgren, Andrea Hinterthuer and Beth Hagman for excellent technical help. This study was supported by Deutsche Forschungsgemeinschaft (Grant KR3344/1-1 to MK).

\section{DISCLOSURE}

The authors have nothing to disclose.

1. Betsholtz C. Insight into the physiological functions of PDGF through genetic studies in mice. Cytokine Growth Factor Rev 2004;15:215-228.

2. Tallquist M, Kazlauskas A. PDGF signaling in cells and mice. Cytokine Growth Factor Rev 2004;15:205-213.

3. Fredriksson L, Li H, Eriksson U. The PDGF family: four gene products form five dimeric isoforms. Cytokine Growth Factor Rev 2004;15:197-204.

4. Heldin $\mathrm{CH}$, Westermark B. Mechanism of action and in vivo role of platelet-derived growth factor. Physiol Rev 1999;79:1283-1316.

5. Leveen P, Pekny M, Gebre-Medhin S, et al. Mice deficient for PDGF B show renal, cardiovascular, and hematological abnormalities. Genes Dev 1994;8:1875-1887.

6. Soriano P. Abnormal kidney development and hematological disorders in PDGF beta-receptor mutant mice. Genes Dev 1994;8:1888-1896.

7. Chiara $\mathrm{F}$, Goumans MJ, Forsberg $\mathrm{H}$, et al. A gain of function mutation in the activation loop of platelet-derived growth factor beta-receptor deregulates its kinase activity. J Biol Chem 2004;279:42516-42527.

8. Bonner JC. Regulation of PDGF and its receptors in fibrotic diseases. Cytokine Growth Factor Rev 2004;15:255-273.

9. Werner S, Grose R. Regulation of wound healing by growth factors and cytokines. Physiol Rev 2003;83:835-870.

10. Heuchel R, Berg A, Tallquist $M$, et al. Platelet-derived growth factor beta receptor regulates interstitial fluid homeostasis through phosphatidylinositol-3' kinase signaling. Proc Natl Acad Sci USA 1999:96:11410-11415.

11. Karlsson S, Kowanetz K, Sandin A, et al. Loss of T-cell protein tyrosine phosphatase induces recycling of the platelet-derived growth factor (PDGF) beta-receptor but not the PDGF alpha-receptor. Mol Biol Cell 2006;17:4846-4855.

12. Weber LW, Boll M, Stampfl A. Hepatotoxicity and mechanism of action of haloalkanes: carbon tetrachloride as a toxicological model. Crit Rev Toxicol 2003;33:105-136.

13. Kappert K, Sparwel J, Sandin A, et al. Antioxidants relieve phosphatase inhibition and reduce PDGF signaling in cultured VSMCs and in restenosis. Arterioscler Thromb Vasc Biol 2006;26:2644-2651.

14. Saito $S$, Frank GD, Mifune $M$, et al. Ligand-independent trans-activation of the platelet-derived growth factor receptor by reactive oxygen species requires protein kinase C-delta and c-Src. J Biol Chem 2002;277:44695-44700.

15. Salmon M, Dedessus Le Moutier J, Wenders F, et al. Role of the PLA2independent peroxiredoxin $\mathrm{VI}$ activity in the survival of immortalized fibroblasts exposed to cytotoxic oxidative stress. FEBS Lett 2004;557:26-32. 
16. Saile B, Ramadori G. Inflammation, damage repair and liver fibrosis-role of cytokines and different cell types. Z Gastroenterol 2007:45:77-86.

17. Ogawa T, Tateno C, Asahina K, et al. Identification of vitamin A-free cells in a stellate cell-enriched fraction of normal rat liver as myofibroblasts. Histochem Cell Biol 2007;127:161-174.

18. Gressner OA, Weiskirchen R, Gressner AM. Evolving concepts of liver fibrogenesis provide new diagnostic and therapeutic options. Comp Hepatol 2007;6:7.

19. Guyot C, Lepreux S, Combe C, et al. Hepatic fibrosis and cirrhosis: the (myo)fibroblastic cell subpopulations involved. Int J Biochem Cell Biol 2006;38:135-151.

20. Ramadori G, Saile B. Portal tract fibrogenesis in the liver. Lab Invest 2004;84:153-159.

21. Wong FW, Chan WY, Lee SS. Resistance to carbon tetrachlorideinduced hepatotoxicity in mice which lack CYP2E1 expression. Toxicol Appl Pharmacol 1998;153:109-118.

22. Marra F, Gentilini A, Pinzani M, et al. Phosphatidylinositol 3-kinase is required for platelet-derived growth factor's actions on hepatic stellate cells. Gastroenterology 1997;112:1297-1306.

23. Jones AV, Cross NC. Oncogenic derivatives of platelet-derived growth factor receptors. Cell Mol Life Sci 2004;61:2912-2923.
24. Gressner AM, Weiskirchen R, Breitkopf $\mathrm{K}$, et al. Roles of TGF-beta in hepatic fibrosis. Front Biosci 2002;7:d793-d807.

25. Borkham-Kamphorst E, Stoll D, Gressner AM, et al. Antisense strategy against PDGF B-chain proves effective in preventing experimental liver fibrogenesis. Biochem Biophys Res Commun 2004;321:413-423.

26. Yoshiji H, Noguchi R, Kuriyama S, et al. Imatinib mesylate (STI571: Gleevec) attenuates liver fibrosis development in rats. Am J Physiol Gastrointest Liver Physiol 2005;288:G907-G913.

27. Neef $M$, Ledermann $M$, Saegesser $H$, et al. Oral imatinib treatment reduces early fibrogenesis but does not prevent progression in the long term. J Hepatol 2006;44:167-175.

28. Darr D, Fridovich I. Free radicals in cutaneous biology. J Invest Dermatol 1994;102:671-675.

29. Thannickal VJ, Fanburg BL. Reactive oxygen species in cell signaling. Am J Physiol Lung Cell Mol Physiol 2000;279: L1005-L1028.

30. Lee JA, Conejero JA, Mason JM, et al. Lentiviral transfection with the PDGF-B gene improves diabetic wound healing. Plast Reconstr Surg 2005;116:532-538.

31. LeGrand EK. Preclinical promise of becaplermin (rhPDGF-BB) in wound healing. Am J Surg 1998;176(2A Suppl):48S-54S. 